\title{
Prognosis of autoimmune thyroid disease associated with hereditary thrombophilia during pregnancy
}

\author{
FLORINA-PAULA PĂULEȚ ${ }^{1}$, NATALIA ȚURCAN ${ }^{1}$, FLORENTINA GHERGHICEANU ${ }^{2}$, \\ ROXANA ELENA BOHÎLT,EA ${ }^{3}$, DRAGOS NEMESCU ${ }^{4}$ and MONICA MIHAELA CÎRSTOIU ${ }^{3}$
}

\begin{abstract}
${ }^{1}$ Department of Obstetrics Gynecology, 'Carol Davila' University of Medicine and Pharmacy Doctoral School, University Emergency Hospital Bucharest, 050098 Bucharest; ${ }^{2}$ Department of Marketing and Medical Technology, 'Carol Davila' University of Medicine and Pharmacy, 050474 Bucharest; ${ }^{3}$ Department of Obstetrics Gynecology,

'Carol Davila' University of Medicine and Pharmacy, University Emergency Hospital Bucharest, 050098 Bucharest;

${ }^{4}$ Department of Obstetrics Gynecology, University of Medicine and Pharmacy 'Grigore T. Popa', 700115 Iasi, Romania
\end{abstract}

Received March 26, 2020; Accepted April 27, 2020

DOI: $10.3892 /$ etm.2020.8785

\begin{abstract}
Autoimmune thyroid pathology has been connected with several systemic autoimmune disorders, namely hereditary thrombophilia and antiphospholipid syndrome. In an iodine-replete country, these diseases need to be evaluated simultaneously. This study was conducted to assess the connection between acute and chronic thyroiditis and inherited thrombophilia and their potential implications in pregnancy. In the analysis of the cases admitted to the Department of Obstetrics Gynecology of the University Emergency Hospital Bucharest from January 2015 up to September 2019, we identified pregnancies marked by autoimmune hypothyroidism and hyperthyroidism associated with hereditary thrombophilia, by standard statistical methods. In this investigation, we determined the prevalence of immunological markers specific for thrombophilia and against thyroid antigens in pregnant women, and explored whether they associate with distinct clinical phenotypes. Besides the well-accounted impact of hereditary thrombophilia during pregnancy - which is emphasized in our study by the incidence of moderate preeclampsia - we also account for a direct and statistically significant relationship between thrombophilia and moderate intensity autoimmune diseases (including autoimmune thyroiditis). Moreover, if these two pathologies are combined there is an increased risk of the presence of another autoimmune disease.
\end{abstract}

Correspondence to: Dr Roxana Elena Bohîlțea, Department of Obstetrics Gynecology, 'Carol Davila' University of Medicine and Pharmacy, University Emergency Hospital Bucharest, 169 Splaiul Independenței, 050098 Bucharest, Romania

E-mail: r.bohiltea@yahoo.com

Key words: autoimmune, inherited, iodine-replete, thrombophilia, thyroid, pregnancy

\section{Introduction}

An autoimmune disease (AID) is marked by tissue damage, inflicted by the self-reactivity of the various mechanisms of the immune system, antibodies and $\mathrm{T}$ cells. Its on-set could be associated with genetic and/or environmental triggers. Although, individually, each AID is present in a small number of individuals, overall, its prevalence is estimated to be between 7.6 and $9.4 \%$ (1). All autoimmune diseases, to some extent, have repercussions for obstetrics and not only. For example, in the general population, $\sim 80 \%$ of the abortions happen during the first 12 weeks of pregnancy, and the risk of spontaneous abortion in women aged $<35$ years is $\sim 10 \%$, while in those aged $>40$ years is exceeding $45 \%(2,3)$. Clinical immunology studies have shown that autoimmune thyroiditis is associated with high rates of infertility and early miscarriages due to associated hormonal changes and instability. The presence of anti-thyroid antibodies may react against the structures of the placenta or fertilized egg and may cause embryo implantation problems (4).

The need for systematic thyroid function and TAb evaluation in early pregnancy may seem fairly obvious, but this is far from being a unanimous opinion $(5,6)$. It is 8 years since we called for thyroid function screening in pregnancy and 5 years since the American Association of Clinical Endocrinologists (AACE) recommended thyroid function screening in all women during the first trimester of pregnancy. The most recent joint guideline published by the Endocrine Society recommends an aggressive case-finding approach during early pregnancy in high-risk populations rather than routine screening.

In the present analysis, data were pooled from the clinical database that spans over 57 months of the Department of Obstetrics Gynecology of the University Emergency Hospital Bucharest, in order to evaluate the connection between AIDs (autoimmune thyroiditis and inherited thrombophilia) in a sufficiently large sample of pregnant women.

\section{Patients and methods}

In the present retrospective analysis, it was aimed to determine the prognosis, regarding fetal and maternal morbidity, 


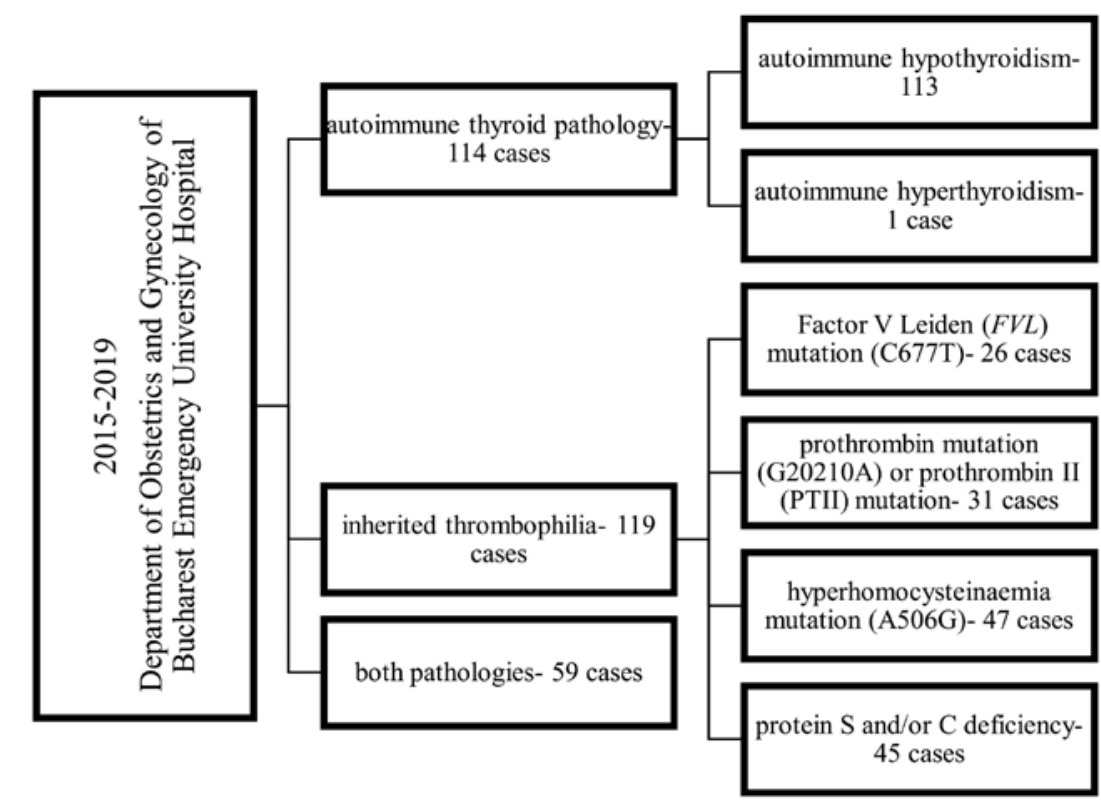

Figure 1. Distribution in pregnant women diagnosed with thyroid pathology and inherited thrombophilia during 57 months in the Department of Obstetrics Gynecology of the University Emergency Hospital Bucharest.

when autoimmune thyroid pathologies associate with inherited thrombophilia, simultaneously throughout the pregnancy. The patients that met our criteria were divided into three groups - pregnancies associating only autoimmune thyroid pathology (mainly autoimmune thyroiditis), pregnancies affected only by inherited thrombophilia, and cases marked by the two diseases simultaneously. The inclucion criteria for the first two groups were based on the presence of a gene mutation such as Factor V Leiden $(F V L)$ mutation (C677T), hyperhomocysteinaemia mutation (A506G), prothrombin mutation (G20210A) or prothrombin II (PTII) mutation, or protein $\mathrm{S}$ and/or $\mathrm{C}$ deficiency detected according to the guidelines of the American College of Obstetricians and Gynecologists Practice Bulletin No. $124(7,8)$ for the first group, and the presence of thyroid antibodies (ATAs) [anti-thyroglobulin (anti-TG) and anti-thyroid peroxidase (anti-TPO)], on two or more occasions, at least 6 weeks apart, for the second group. Thus, the studied population consisted of 82 pregnancies marked only by autoimmune thyroid pathology, 65 pregnancies affected by inherited thrombophilia, and a total of 27 pregnancies that associated both diseases (Fig. 1). The patients were followed at the outpatient clinic of the Department of Obstetrics Gynecology, University Emergency Hospital Bucharest. Informed consent was obtained from all patients. Patients' medical charts were retrospectively evaluated for clinical and laboratory data. Clinical data collection was based on a standardized interview completed by all patients presenting with features of autoimmune diseases.

In the first stage of statistical evaluation, descriptive tests were applied while monitoring the presence of risk factors and complications in the studied groups. The main parameters analyzed were the fetal weight at birth and Apgar score (to assess the impact on the fetal outcome) - Figs. 2 and 3, as well as the risk of developing pre-eclampsia, intrauterine growth restriction, diabetes, abruption placentae and premature birth.
The frequency for each studied group and their comparative reproduction is shown in Fig. 4.

\section{Results}

In the analysis conducted above, of the cases admitted to the Department of Obstetrics Gynecology of the University Emergency Hospital Bucharest from January 2015 up to September 2019, we identified 171 pregnancies marked by hypothyroidism and 8 cases with hyperthyroidism.

Twenty-seven cases of autoimmune thyroiditis also associated hereditary thrombophilia, compared with one case of hyperthyroidism (Fig. 1). This is also reflective of the fact that Romania belongs to an iodine-replete area.

Regarding the comparative analysis of the three studied groups and the implications of the studied pathologies in pregnancy a significant and direct statistical significant relation between thrombophilia and mild preeclampsia were obtained, $\mathrm{P}=0.007(<0.05)$, Cramer $\mathrm{V}=0.19$; OR, 10.23; 95\% CI, 1.22-85.79.

There is a significant statistical correlation between the presence of thrombophilia and autoimmune thyroiditis, $\mathrm{P}=0.004$, Cramer $\mathrm{V}=0.20$; OR, 4.57; 95\% CI, 1.46-14.27. Continuing this context, based on our statistical analysis if a patient is associated with thrombophilia and autoimmune thyroiditis the risk for the presence of another autoimmune disease is increased 3.86-fold, $\mathrm{P}=0.008$, Cramer $\mathrm{V}=0.19$; OR, 3.86; 95\% CI, 1.36-10.94.

We obtained significant results by comparative analysis of group 1 with group 3 , respectively, patients with only autoimmune thyroid pathology (mainly autoimmune thyroiditis) and patients with inherited thrombophilia and autoimmune thyroid pathology for the incidence of premature birth, which appears with a significantly increased incidence in patients associating both pathologies. Gestational diabetes also appears with a significantly increased incidence in group 3 . 


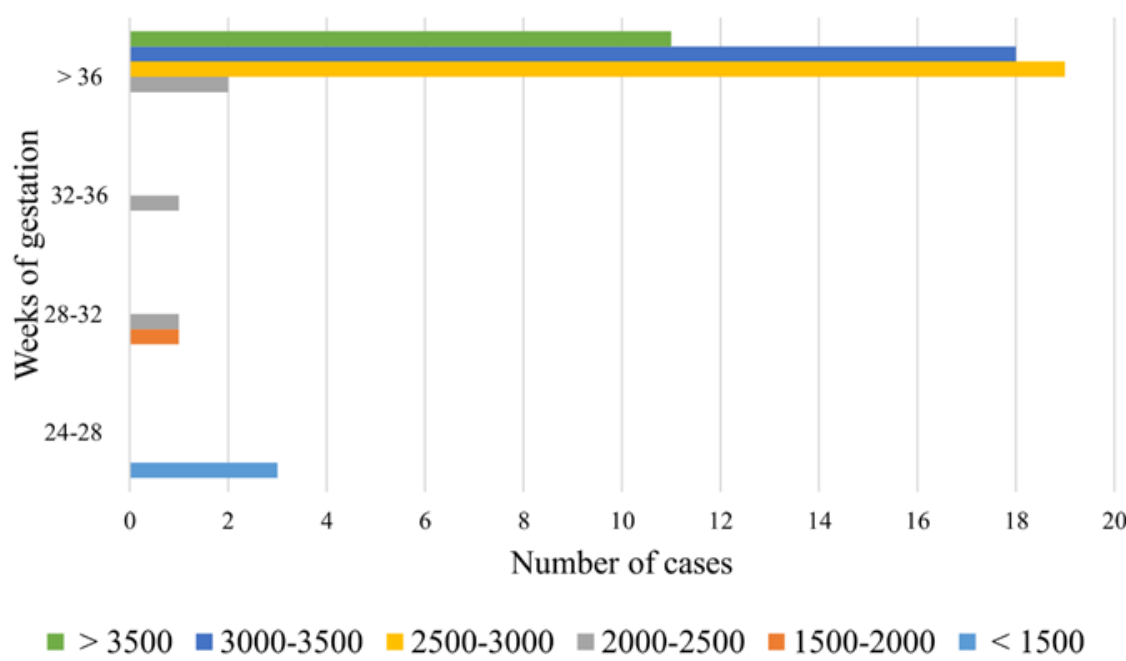

Figure 2. Distribution of fetal gestational age and weight at birth from mothers suffering of autoimmune thyroid pathology in the Department of Obstetrics Gynecology of the University Emergency Hospital Bucharest. Study duration 57 months.

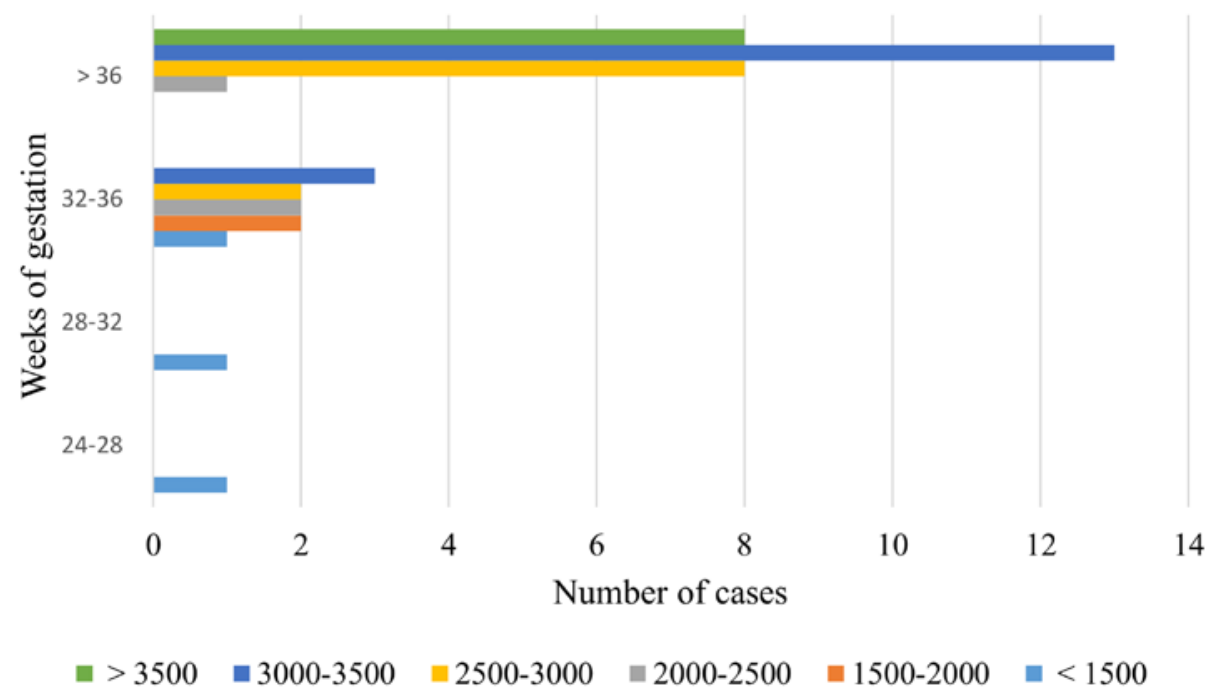

Figure 3. Distribution of fetal gestational age and weight at birth from mothers suffering of autoimmune thyroid pathology and inherited thrombophilia in the Department of Obstetrics Gynecology of the University Emergency Hospital Bucharest. Study duration 57 months.
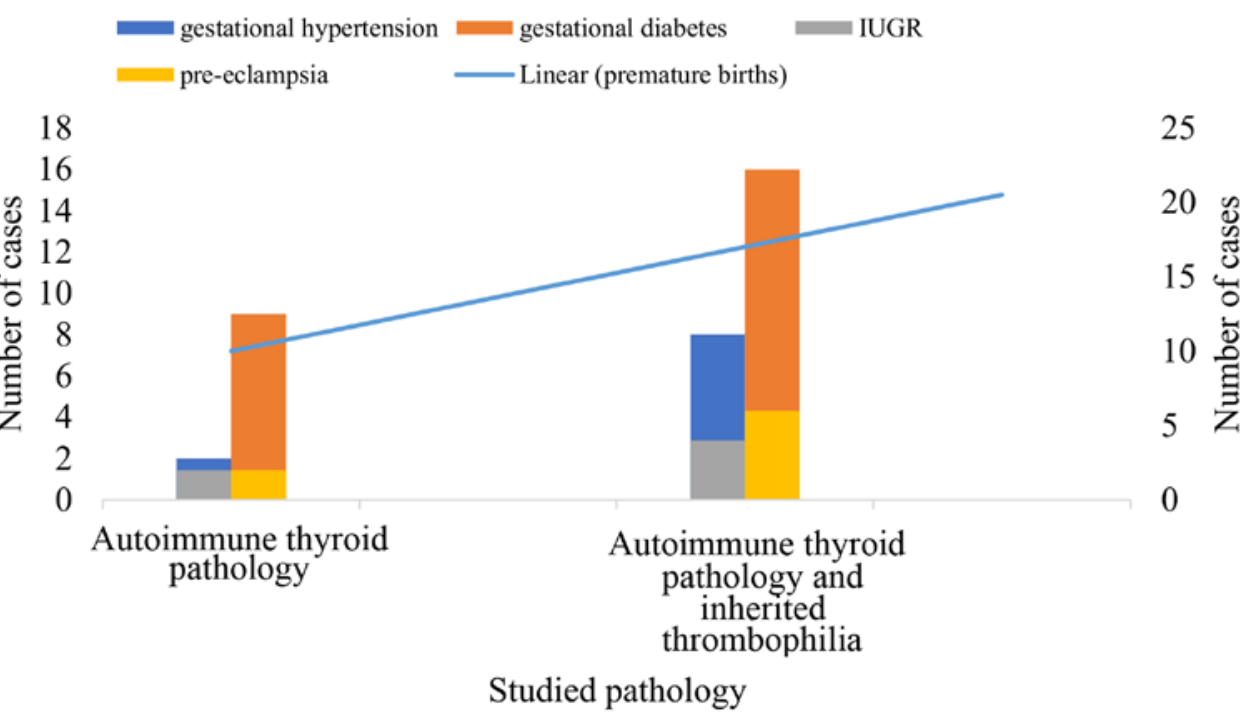

Figure 4. Distribution in pregnant women diagnosed with complications during pregnancies associating autoimmune thyroid pathology and inherited thrombophilia in the Department of Obstetrics Gynecology of the University Emergency Hospital Bucharest. Study duration 57 months. 


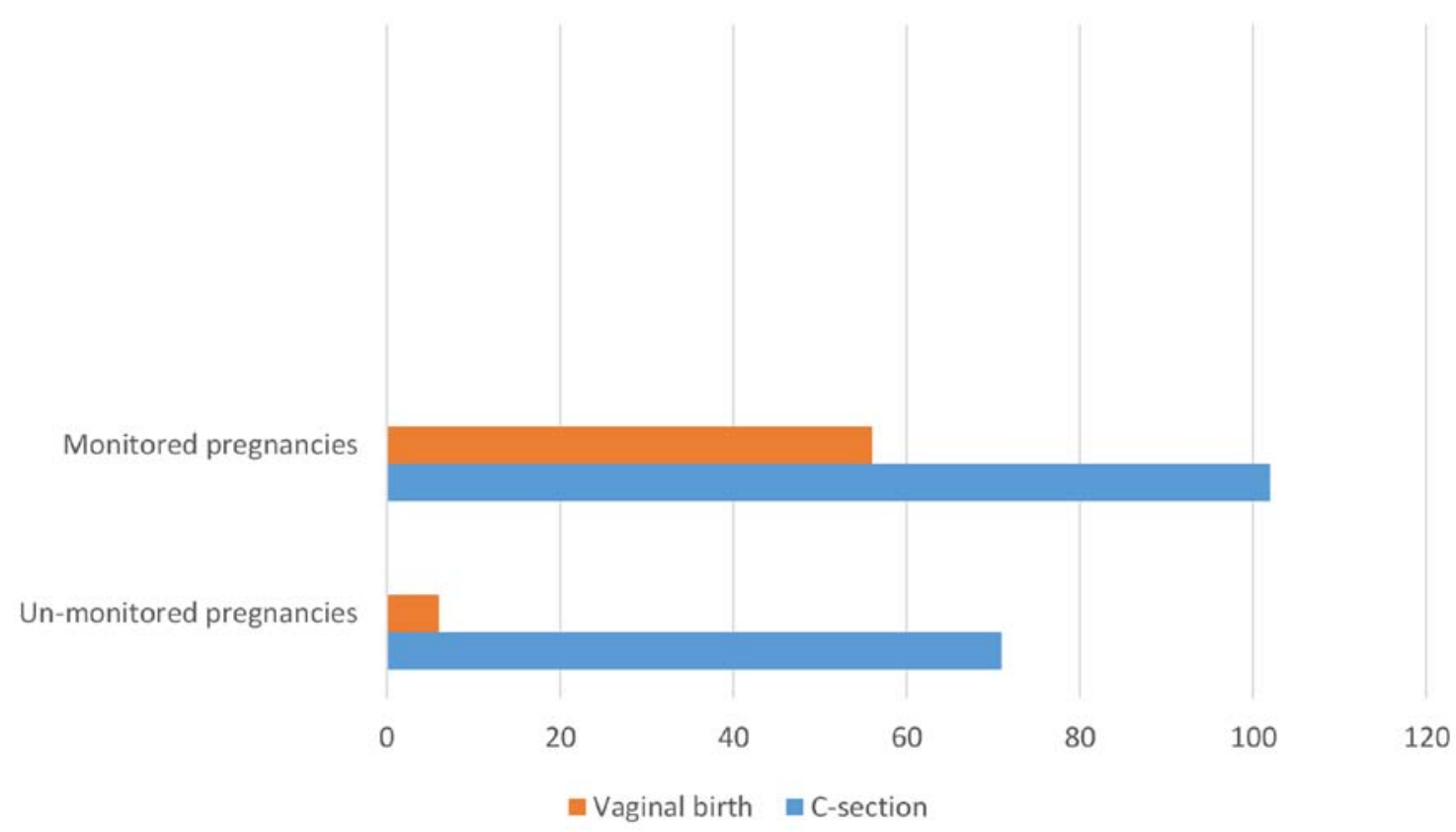

Figure 5. Comparison between C-section and vaginal birth of monitored and un-monitored pregnancies associating autoimmune thyroid pathology and hereditary thrombophilia during 57 months in the Department of Obstetrics Gynecology of the University Emergency Hospital Bucharest.

Consequently, we also observed that out of the 27 pregnancies associating thyroid pathology and inherited thrombophilia, in 24 cases the pregnancy was not monitored by any kind of health care provider (Fig. 5). This is a major factor in a developing country, and a continuous source of monetary loss for our health care system.

\section{Discussion}

Regarding the statistics of the two pathologies affecting the pregnant woman, the data collected in Romania is scarce. The prevalence and incidence of thyroid dysfunction are difficult to compare across countries due to differences in diagnostic thresholds, assay sensitivities, population selection and fluxes in iodine nutrition and population dynamics.

A meta-analysis of European studies estimated a mean prevalence rate for autoimmune thyroiditis of $0.75 \%$ for males and females combined and an incidence rate of 51 cases per 100,000 per year (9). However, a database for this pathology associated with pregnancy is not yet available. Thyrotoxicosis in pregnancy has an estimated incidence of $0.2 \%$ for overt thyrotoxicosis and $2.5 \%$ for subclinical thyrotoxicosis (10-12).

Iodine deficiency and autoimmune disease (Hashimoto thyroiditis) are responsible for the majority of cases of primary hypothyroidism (13). A third of the world's population lives in iodine-deficient areas, and the devastating consequences of severe iodine deficiency on the neurological development of fetuses and children are well recognized. In addition, the possible effects of less severe grades of iodine deficiency during pregnancy on offspring cognitive development are also becoming recognized (14). In Europe, $44 \%$ of school-age children still have insufficient iodine intake. The prevalence of overt hypothyroidism in the general population ranges from between 0.2 and $5.3 \%$ in Europe $(15,16)$.
A point of discussion worth pursuing is the link between autoimmune thyroid pathology and pregnancy loss. Autoimmune factors seem to play a pivotal role in infertility. The prevalence of antiphospholipid antibodies (aPL), ANA, and antithyroid antibodies was found to be particularly higher in women with unknown infertility. Implantation is a process based on immunologic tolerance and requires cross-talk between the embryo and the maternal immune system. Impaired implantation and high pregnancy loss rates in women with autoimmune antibodies could be caused by injury to the syncytiotrophoblasts, endothelial cells of the spiral veins, superficial/glandular epithelial cells of the decidua or autoantibody inflammatory processes. These biological events result in impaired implantation and disturbed fetal perfusion (17). During the analysis presented above, we also observed 25 cases of autoimmune hypothyroidism associated with spontaneous abortions at gestational ages ranging between 8 and 22 weeks, out of which 4 cases also suffered from inherited thrombophilia. Although exact screening and management protocols for infertile patients positive for autoimmune antibodies have not been established, it seems reasonable to devise a patient-centered, individualized approach for these patients.

The present analysis had several limitations. Due to financial and geographic limitations, a few of the pregnant women did not report to the outpatient clinic regularly, in order to be tested. After giving birth, some of the patients did not return to the 6-week check-up, in order to evaluate their state of health outside the stress of the pregnancy. We did not pursue the implications of the AIDs during pregnancy on the foetus (weight, neurological and morphological features). Furthermore, the small number of patients, the retrospective design of the study, and that it reflects the experience of a single center. However, this study was designed to stress the importance of knowing the immunological background of the pregnant woman. 
This analysis pointed to the need to consider also testing for other AIDs such as antiphospholipid syndrome. Although the presence of antiphospholipid antibodies in patients with autoimmune thyroiditis has been previously reported (18), only a small study highlights the prevalence of antibodies against thyroid antigens in patients with antiphospholipid syndrome (APS). Moreover, the increasingly recognized association of thyroid autoimmunity with central nervous system (CNS) disease and pregnancy morbidity features, which can both be found in the APS (19), prompts us to further investigate the connection between thyroid autoimmunity, APS and pregnancy. In addition, continued vigilance against iodine deficiency remains essential in developed countries, particularly in Europe. Universal thyroid screening associated with thrombophilia screening in pregnancy is therefore contentious in iodine-replete areas, although it has been shown to be cost-effective in analytical economic models.

Besides the well-accounted impact of hereditary thrombophilia during pregnancy, which is emphasized in our study by the incidence of moderate preeclampsia, we also account for a direct and statistically significant relationship between thrombophilia and moderate intensity autoimmune diseases (including autoimmune thyroiditis). Furthermore, if these two pathologies are combined there is an increased risk of the presence of another autoimmune disease.

\section{Acknowledgements}

Not applicable.

\section{Funding}

No funding was received.

\section{Availability of data and materials}

The datasets used and/or analyzed during the current study are available from the corresponding author on reasonable request.

\section{Authors' contributions}

FPP, REB and NT collected, analyzed and interpreted the patient data regarding the testing for ATAs, anti-TG, anti-TPO, TSH, freeT 3 and freeT $_{4}$. FPP, REB and NT also collected the data of immunological and genetic markers specific for thrombophilia and antiphospholipid syndrome of the pregnant women included in the present study. FG, DN and MMC substantially contributed to the conception of the study, the interpretation of the data and the writing of the manuscript. All authors read and approved the final version of the manuscript.

\section{Ethics approval and consent to participate}

Informed consent was obtained from all patients.

\section{Patient consent for publication}

\section{Not applicable.}

\section{Competing interests}

The authors declare that they have no competing interests.

\section{References}

1. Cooper GS, Bynum ML and Somers EC: Recent insights in the epidemiology of autoimmune diseases: Improved prevalence estimates and understanding of clustering of diseases. J Autoimmun 33: 197-207, 2009.

2. Hurt KJ, Guile MW, Bienstock JL, Fox HE and Wallach EE (eds): The Johns Hopkins Manual of Gynecology and Obstetrics (Lippincott Manual Series). 4th, North American edition. Lippincott Williams and Wilkins, Philadelphia, PA, pp73-77, 2011.

3. Pearce EN, Andersson M and Zimmermann MB: Global iodine nutrition: Where do we stand in 2013? Thyroid 23: 523-528, 2013.

4. Grassi G, Balsamo A, Ansaldi C, Balbo A, Massobrio M and Benedetto C: Thyroid autoimmunity and infertility. Gynecol Endocrinol 15: 389-396, 2001.

5. Abalovich M, Amino N, Barbour LA, Cobin RH, De Groot LJ, Glinoer D, Mandel SJ and Stagnaro-Green A: Management of thyroid dysfunction during pregnancy and postpartum: An Endocrine Society Clinical Practice Guideline. J Clin Endocrinol Metab 92 (Suppl): S1-S47, 2007.

6. American College of Obstetrics and Gynecology: ACOG practice bulletin. Thyroid disease in pregnancy. Number 37, August 2002. Int J Gynaecol Obstet 79: 171-180, 2002.

7. Negro R, Formoso G, Mangieri T, Pezzarossa A, Dazzi D and Hassan H: Levothyroxine treatment in euthyroid pregnant women with autoimmune thyroid disease: Effects on obstetrical complications. J Clin Endocrinol Metab 91: 2587-2591, 2006.

8. Lockwood C and Wendel G; Committee on Practice Bulletins Obstetrics: Practice bulletin no. 124: Inherited thrombophilias in pregnancy. Obstet Gynecol 118: 730-740, 2011.

9. Stevens SM, Woller SC, Bauer KA, Kasthuri R, Cushman M, Streiff M, Lim W and Douketis JD: Guidance for the evaluation and treatment of hereditary and acquired thrombophilia. J Thromb Thrombolysis 41: 154-164, 2016.

10. Innocencio RM, Romaldini JH and Ward LS: High prevalence of thyroid autoantibodies in systemic sclerosis and rheumatoid arthritis but not in the antiphospholipid syndrome. Clin Rheumatol 22: 494, 2003.

11. Garmendia Madariaga A, Santos Palacios S, Guillén-Grima F and Galofré JC: The incidence and prevalence of thyroid dysfunction in Europe: A meta-analysis. J Clin Endocrinol Metab 99: 923-931, 2014.

12. Turcan N, Bohîlţea R, Neacsu A, Baros A and Cîrstoiu MM: The role of anticoagulant therapy in the prevention of preeclampsia pharmacokinetic and pharmacodynamic mechanisms. Rev Chim 70: 1424-1428, 2019.

13. Asvold BO, Vatten LJ and Bjøro T: Changes in the prevalence of hypothyroidism: The HUNT Study in Norway. Eur J Endocrinol 169: 613-620, 2013.

14. Bohîltea RE, Zugravu CA, Neacsu A, Navolan D, Berceanu C, Nemescu D, Bodean O, Turcan N, Baros A and Cîrstoiu MM: The prevalence of vitamin D defficiency and its obstetrical effects. A prospective study on Romanian patients. Rev Chim 70: 1228-1233, 2019.

15. Okosieme OE and Lazarus JH: Important considerations in the management of Graves' disease in pregnant women. Expert Rev Clin Immunol 11: 947-957, 2015.

16. Taylor PN and Vaidya B: Side effects of anti-thyroid drugs and their impact on the choice of treatment for thyrotoxicosis in pregnancy. Eur Thyroid J 1: 176-185, 2012.

17. Chaker L, Bianco AC, Jonklaas J and Peeters RP: Hypothyroidism. Lancet 390: 1550-1562, 2017.

18. Bohîltea R, Turcan N, Ionescu C, Toader O, Nastasia S, Neculcea D, Movileanu I, Munteanu O and Cîrstoiu M: The incidence of prematurity and associated short-term complications in a multidisciplinary emergency hospital from Romania. In: Proceedings of the 5th Congress of the Romanian Society of Ultrasound in Obstetrics and Gynecology, Filodiritto Editore, pp105-112, 2017.

19. Nabriski D, Ellis M, Ness-Abramof R, Shapiro M and Shenkman L: Autoimmune thyroid disease and antiphospholipid antibodies. Am J Hematol 64: 73-75, 2000. 